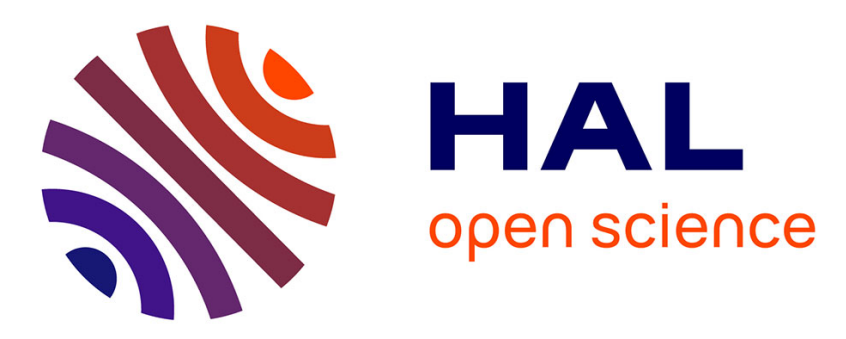

\title{
Fully Automated Electrophysiological Model Personalisation Framework from CT Imaging
}

Nicolas Cedilnik, Josselin Duchateau, Frederic Sacher, Pierre Jaïs, Hubert Cochet, Maxime Sermesant

\section{- To cite this version:}

Nicolas Cedilnik, Josselin Duchateau, Frederic Sacher, Pierre Jaïs, Hubert Cochet, et al.. Fully Automated Electrophysiological Model Personalisation Framework from CT Imaging. FIMH 2019 - 10th International Conference on Functional Imaging and Modeling of the Heart, Jun 2019, Bordeaux, France. pp.325-333. hal-02106609

\section{HAL Id: hal-02106609 https://hal.inria.fr/hal-02106609}

Submitted on 23 Apr 2019

HAL is a multi-disciplinary open access archive for the deposit and dissemination of scientific research documents, whether they are published or not. The documents may come from teaching and research institutions in France or abroad, or from public or private research centers.
L'archive ouverte pluridisciplinaire HAL, est destinée au dépôt et à la diffusion de documents scientifiques de niveau recherche, publiés ou non, émanant des établissements d'enseignement et de recherche français ou étrangers, des laboratoires publics ou privés. 


\title{
Fully Automated Electrophysiological Model Personalisation Framework from CT Imaging
}

\author{
Nicolas Cedilnik ${ }^{1,2}$, Josselin Duchateau ${ }^{2}$, Frédéric Sacher ${ }^{2}$, Pierre Jaïs ${ }^{2}$, Hubert \\ Cochet $^{2}$, and Maxime Sermesant ${ }^{1}$ \\ 1 Université Côte d'Azur, Epione Research Project, Inria, Sophia Antipolis \\ 2 Liryc Institute, Bordeaux
}

\begin{abstract}
There has been a recent growing interest for cardiac computed tomography (CT) imaging in the electrophysiological community. This imaging modality indeed allows to locate and assess post-infarct scar heterogeneity, allowing to predict zones of abnormal electrical activity and even personalise EP models.

To this end, most of the literature uses manually segmented CT images where one fundamental information is extracted, the myocardial wall thickness. In this paper, we evaluate the impact of using an automated deep learning (DL) methodology to segment the left ventricular wall and extract relevant scar information on the resulting personalised models. Using CT images from 8 patients that were not used during the DL training, we show that the automated segmentation is very similar to the manual one (median Dice score: 0.9). Thickness information obtained this way is also very close to the manual one (median difference: $0.7 \mathrm{~mm}$ ). A wavefront propagation model personalisation framework based on this thickness information does not show relevant differences in its output (median difference in local activation time: $2 \mathrm{~ms}$ ), proving its robustness. Bipolar electrograms, simulated through a novel approach, do not differ significantly between manual and automated segmentations (Pearson's $r: 0.99)$.
\end{abstract}

Keywords: Imaging $\cdot$ Deep learning $\cdot$ Segmentation $\cdot$ Model Personalisation

\section{Introduction}

Catheter radiofrequency ablation of the ischemic arrhythmogenic substrate has been shown to be efficient to prevent sudden cardiac deaths. This efficiency can partially be attributed to the integration of information extracted from cardiac imaging data both prior to and during the intervention [11.

While cardiac magnetic resonance imaging (MRI) is still widely considered the gold standard for ischemic scar assessment and model personalisation 9, computed tomography $(\mathrm{CT})$ has recently gained interest in the electrophysiological (EP) community. Cardiac CT is indeed able to locate the chronic ischemic scar and evaluate its heterogeneity [7/4]. This is possible by identifying zones of myocardial wall thinning and by evaluating the severity of this thinning; this 


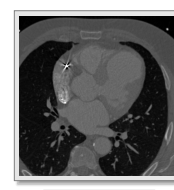

Original CT

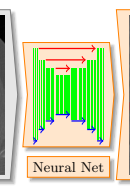

LV masks

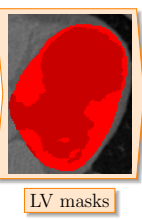

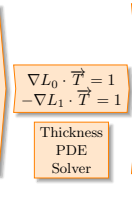
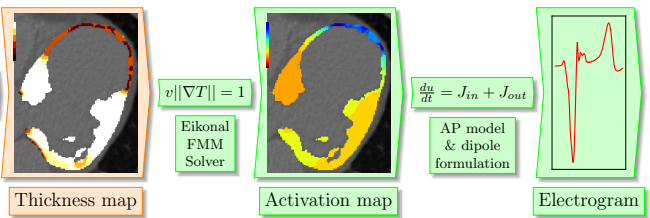

Fig. 1. The model personalisation pipeline. Orange: image processing steps; Green: modelling steps

approach is even able to predict abnormal electrical activity [10|2. Moreover, $\mathrm{CT}$ is less affected than MRI by the presence of a implantable cardioverter defibrillator, a device commonly found in patients susceptible to undergo catheter ablation of ventricular tachycardia.

Given these scar characteristics on CT images, it is no surprise that CT has been successfully used as a way to personalise EP models [1]. However, up until now, this personalisation relies on manual or semi-automated segmentation of the left ventricular (LV) wall, despite the availability of efficient three-dimensional medical image automated segmentation methods $[5]$.

In this paper we evaluate the impact of a deep learning automated segmentation approach on CT ischemic scar assessment and the robustness of the related model personalisation framework. This framework (Figure 1) takes a cardiac CT image as input where the LV is automatically segmented using a neural network, allowing myocardial thickness to be automatically computed [12. After choosing a virtual pacing point, activation maps can be simulated using the Eikonal model. Finally, electrograms can be generated through a novel approach presented in subsection 2.4 .

\section{Methods}

\subsection{Deep Learning Segmentation}

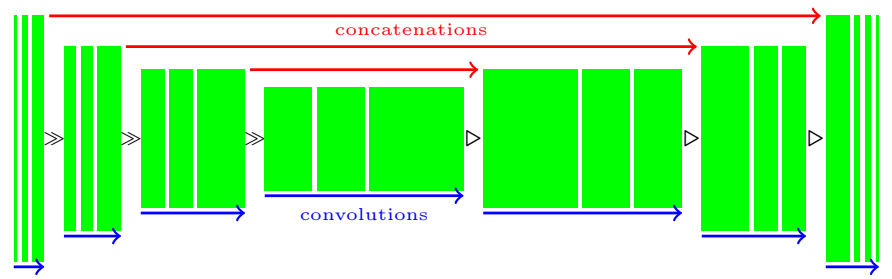

Fig. 2. Architecture of the U-net used to segment cardiac CT images (green blocks: 3D

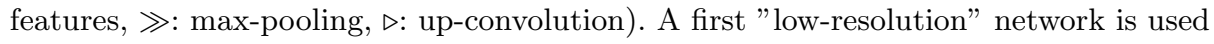
to determine the left ventricle location on the original CT image. A cropped version of the image is then fed to a "high resolution" net. Adapted from [6]. 
The deep learning approach we applied is based on a previously described methodology to segment the left atrium [ 6 . It relies on the use of two successive specialised U-nets [5]. The first is used to coarsely segment the full original CT image. Its output is used to compute the bounding box of the region of interest. This allows a cropping of the original image for a higher resolution segmentation of the desired structures.

Database and Training The network was trained from scratch using a database of 500 cardiac manual segmentations of contrast-enhanced CT images. These segmentations comprise the LV endocardium, the LV epicardium and the right ventricular epicardium. We used 450 cases for training per se and 50 cases for validation with a loss function defined as the opposite of a label-wise Dice score. The model was fitted using an nVidia GeForce $1080 \mathrm{Ti}$ provided by the NEF computing platform.

"Low Resolution" U-Net The first network's input is the original CT image resampled to $128 \times 128 \times 128$ voxels and it outputs 3 ventricular masks: 2 for the left ventricle (epicardium and endocardium), 1 epicardial right ventricle. The training data was augmented twice, by 2 random rotation of the original image along each axis in the $\left[-\frac{\pi}{8} ; \frac{\pi}{8}\right]$ interval.

"High Resolution" U-Net The second network was trained using cropped $\mathrm{CT}$ images around the LV, with $5 \mathrm{~mm}$ margins and resampled to $144 \times 144 \times 144$ voxels. Each original image was augmented 20 times: by random rotations in the $\left[-\frac{\pi}{7} ; \frac{\pi}{7}\right]$ range along each axis, a random shearing in the $[-0.1 ; 0.1]$ range along each axis and the application of Gaussian blur with a kernel using a random standard deviation picked in the $[0.5 ; 2]$ range for half of the augmentations. The network outputs are the two left ventricular masks.

Post-processing The network's outputs were thresholded at 0.5 to obtain binary $3 \mathrm{D}$ masks. In order to obtain spatially coherent masks, they were filtered to keep only the largest connected component and to forbid overlap between masks. Remaining holes in the masks were filled using the most frequent label in the hole neighbourhood. The masks were finally up-sampled to the original $\mathrm{CT}$ image resolution using a nearest neighbour interpolation.

\subsection{Thickness Computation}

Smooth and robust LV wall thickness estimation is not trivial, especially in three dimensions. It indeed requires to solve a partial differential equation using the endocardium and epicardum masks [12]. Such approach, which assign a thickness value to each voxel of the LV wall mask, is particularly adapted for simulations on regular grids; it has been previously used to such ends [1]. 


\subsection{Electrophysiological Model}

We used the thickness information to parameterise an Eikonal model previously described in [1]. Briefly, wall thinning is related to a macroscopic slowing of the activation front, due to a microscopic zig-zag course of activation in the infarcted tissue. A random pacing point was chosen in the healthy tissue, defined by a wall thickness superior to $5 \mathrm{~mm}$, to initiate the propagation. The simulation was stopped at $500 \mathrm{~ms}$.

\subsection{Electrogram Simulation with the Eikonal Model}

We propose an efficient way to simulate electrograms with the Eikonal model. It couples the activation map with a transmembrane action potential model and a propagation methodology based on the dipole formulation [3]. In this framework, every voxel is a considered a dipole with local current density:

$$
\mathbf{j}_{e q}=-\sigma \nabla v,
$$

where $\sigma$ is the local conductivity and $\nabla v$ is the spatial gradient of the potential $v$. Using the chain rule, we can rewrite (1) as:

$$
\mathbf{j}_{e q}=-\sigma \frac{\partial v}{\partial T} \times \frac{\partial T}{\partial X}=-\sigma \frac{\partial v}{\partial T} \nabla T
$$

where $\nabla T$ is the gradient of the activation map (output of the Eikonal model).

To compute $\frac{\partial v}{\partial T}$, we used a forward Euler scheme to solve the MitchellSchaeffer cardiomyocite action potential model $[8]$ and stored its time derivative. We matched the activation time obtained with this model to the activation time obtained with the Eikonal model. To simulate bipolar electrograms, we placed two virtual electrodes (distance between them: $0.9 \mathrm{~mm}$ ) inside the heart cavity and subtracted one signal to the other.

In order to handle the activation map gradient values at the mask boundaries, we ignored the gradient component(s) in the direction(s) flowing out of the mask. We adapted the volume used in the dipole moment computation accordingly [3].

\subsection{Evaluation of the Automated Segmentation Impact}

To evaluate the automated segmentation impact, we focused on 8 cardiac CT images (and their corresponding manual segmentation) of patients suffering of re-entrant VT. Theses images have been used neither for the automated segmentation training nor its validation. For 6 cases (patients 1-6) the original CT images were available; for the remaining 2 cases, resampled images aligned to the heart short axis were used.

Binary masks were resampled to the original CT image resolution when available, in order to compute a Dice score on the LV wall.

To compare thickness and activation maps, a mid-wall mesh was generated from the manual wall segmentation. Maps corresponding to manual and automated segmentation were then projected on this common frame of reference. A 
point-wise comparison was made possible this way, and median differences were computed.

To compare the electrograms obtained with both segmentation methods, they were compared with a Pearson correlation coefficient $r$.

\section{Results}

\subsection{Segmentation}
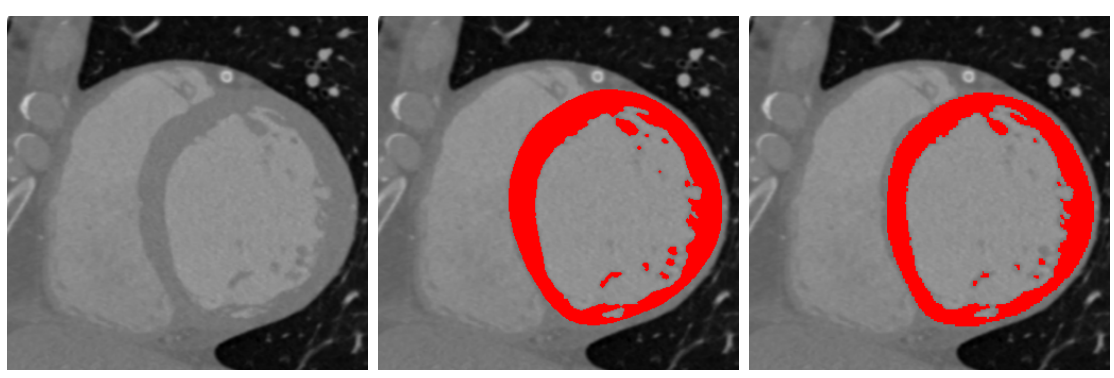

Fig. 3. Visual comparison of manual (middle) and automated (right) segmentations of the left ventricular wall shown on one slice of a CT that was not used during the DL network's fitting.

The training phase of the "high resolution" network reached a plateau at a Dice score of 0.96 for the LV wall. Data augmentation was key to reaching this high score, especially adding the blur filter.

The median Dice score of the 8 images after up-sampling was lower: 0.90 . There is no notable difference in the segmentation quality when using original $\mathrm{CT}$ images versus short-axis resampled images.

In zones of extreme thinning, the wall continuity was not always observed.

\subsection{Thickness Computation}

Across all cases, the median thickness of the manually segmented walls was $5.8 \pm 3.2 \mathrm{~mm}$ versus $5.8 \pm 3.0 \mathrm{~mm}$ for the automatic segmentation. Very similar thickness maps were obtained using the automated segmentation (median differences across all cases: $0.7 \mathrm{~mm}$, see Figure 4).

\subsection{Eikonal Model}

As expected, given similar thickness maps and geometries, the "virtual pacing" results were very close. The median activation time of the manual segmentation models was $143 \mathrm{~ms}$, with a median difference with automated segmentation as little as $2 \mathrm{~ms}$ across all cases. 
N. Cedilnik et al.
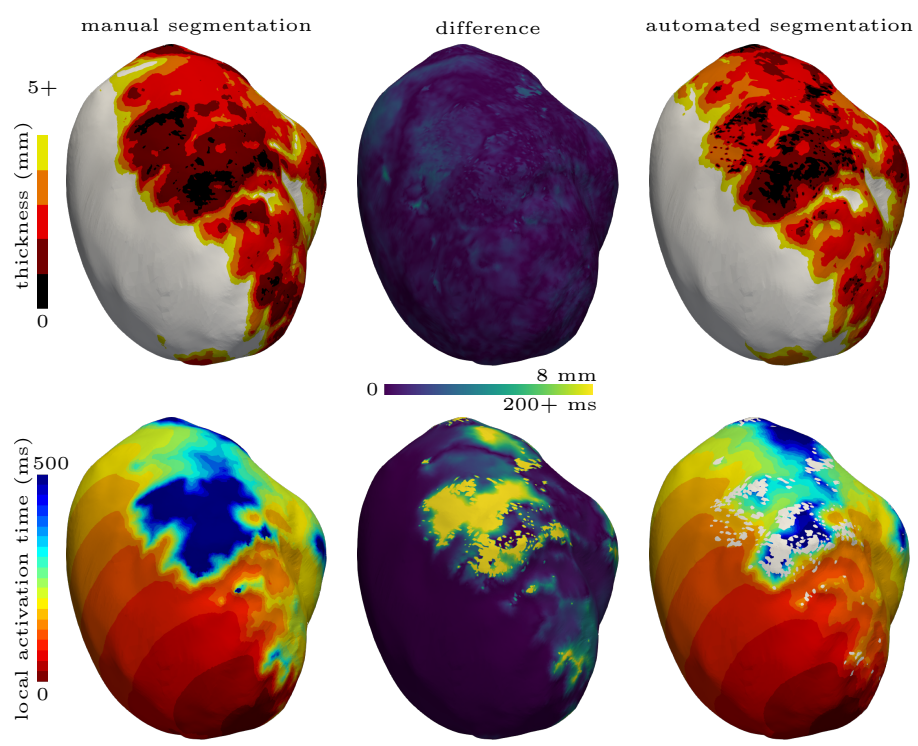

Fig. 4. Impact of the automated segmentation on the thickness and activation maps for one example patient. The values are projected on the mid-wall meshes that were used for quantitative comparisons.

\subsection{Electrogram Simulations}

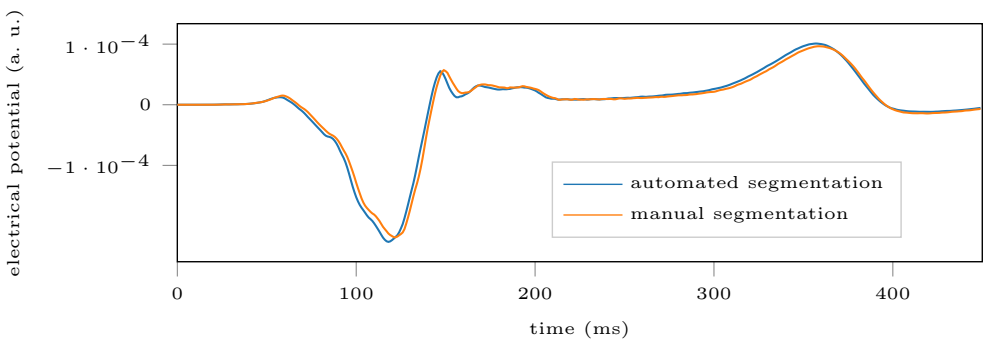

Fig. 5. Example comparison between simulated intra-cardiac bipolar electrograms using manual and automated segmentations (see subsection 2.4 for the methodology)

Bipolar signals generated with the automated and manual segmentations were virtually identical, with a median Pearson's $r$ correlation coefficient of 0.99 . All $r$ s were above 0.94 except one (patient 8, 0.75), and all had $p$-values below $10^{-10}$. 
Table 1. [Left] Segmentation quality. DCS: dice score; HD: Hausdorff Distance; ADH: Average Hausdorff Distance; TD: Thickness Median Difference. [Right] Model robustness: difference between outputs from manual and automated segmentation. LATD: Local Activation Time Median Difference; EGr: Pearson's $r$ coefficient between electrograms $\left(p<10^{-10}\right.$ for all patients)

\begin{tabular}{l|llll|ll} 
Patient & DSC & HD $(\mathrm{mm})$ & AHD $(\mathrm{mm})$ & TD $(\mathrm{mm})$ & LATD $(\mathrm{ms})$ & EGr \\
\hline 1 & 0.88 & 19.15 & 0.10 & 0.7 & 5 & 0.99 \\
4 & 0.90 & 17.47 & 0.09 & 0.8 & 2 & 0.99 \\
5 & 0.87 & 13.25 & 0.09 & 0.8 & 3 & 0.99 \\
6 & 0.91 & 5.00 & 0.05 & 0.5 & 1 & 1.00 \\
7 & 0.91 & 16.44 & 0.06 & 0.6 & 1 & 1.00 \\
8 & 0.88 & 7.57 & 0.09 & 1.4 & 29 & 0.75 \\
9 & 0.89 & 22.86 & 0.11 & 0.7 & 2 & 0.94 \\
10 & 0.91 & 12.71 & 0.08 & 0.7 & 1 & 0.99 \\
\hline median & $\mathbf{0 . 9 0}$ & $\mathbf{1 4 . 8 5}$ & $\mathbf{0 . 0 9}$ & $\mathbf{0 . 7}$ & $\mathbf{2}$ & $\mathbf{0 . 9 9}$
\end{tabular}

\section{Discussion}

\subsection{Segmentation}

The automated segmentation algorithm was shown to produce segmentations very close to those obtained by trained radiologists, even with different orientations of the input images. A perfect match between the algorithm and expert segmentation is not desirable anyway as there is also uncertainty in the manual segmentation. The available automated segmentation can probably be improved by training the neural networks on GPUs with more RAM, allowing a better input resolution and less loss of information in the down-sampling phase.

\subsection{Thickness}

The differences in the corresponding thickness maps are even smaller. Scar heterogeneity is preserved and comparable between manual and automated segmentation. In some particular cases of wall configuration the thickness computation could be problematic but it only happens in very localised cases which were easy to identify.

Ideally it would have been preferable to compare scar localization on MRI images, but they were not available for these patients.

\subsection{Modelling}

As expected, similarity of thickness maps leads to very similar simulation output between the automated and manual segmentation. The wall discontinuities are not problematic at all since they concern zones of extreme wall thinning that are considered non conductive anyway. These explain most of the discrepancy between the resulting activation maps. 


\section{Conclusion}

We presented in this manuscript the automatic segmentation of the myocardial wall in CT images and the quantification of its impact on personalised models. We showed that most of the infarct related arrhythmia information extraction from CT images are not affected much by using an automated segmentation methodology, proving its robustness. This is another major step towards the future use of EP model personalisation in clinical practice.

Furthermore, we presented a novel methodology to generate electrograms from activation maps using the Eikonal model and the dipole formulation. Here we used the same action potential characteristics across the whole domain, but our formulation makes it possible to easily vary them, using the LV wall thickness for instance. Filtering the signals obtained in the same way they are filtered in the EP lab could further improve their realism.

\section{References}

1. Cedilnik, N., Duchateau, J., Dubois, R., Sacher, F., Jaïs, P., Cochet, H., Sermesant, M.: Fast Personalized Electrophysiological Models from CT Images for Ventricular Tachycardia Ablation Planning. EP-Europace 20 (Nov 2018)

2. Ghannam, M., Cochet, H., Jais, P., Sermesant, M., Patel, S., Siontis, K.C., Morady, F., Bogun, F.: Correlation between computer tomography-derived scar topography and critical ablation sites in postinfarction ventricular tachycardia. Journal of Cardiovascular Electrophysiology 29(3) (Jan 2018)

3. Giffard-Roisin, S., Fovargue, L., Webb, J., Molléro, R., Lee, J., Delingette, H., Ayache, N., Razavi, R., Sermesant, M.: Estimation of Purkinje Activation from ECG: An Intermittent Left Bundle Branch Block Study. In: Statistical Atlases and Computational Models of the Heart. Imaging and Modelling Challenges. pp. 135-142. Lecture Notes in Computer Science, Springer, Cham (Oct 2016)

4. Grutta, L.L., Toia, P., Maffei, E., Cademartiri, F., Lagalla, R., Midiri, M.: Infarct characterization using CT. Cardiovascular Diagnosis and Therapy 7(2), 171-188 (Jul 2017)

5. Isensee, F., Kickingereder, P., Wick, W., Bendszus, M., Maier-Hein, K.H.: Brain Tumor Segmentation and Radiomics Survival Prediction: Contribution to the BRATS 2017 Challenge. arXiv:1802.10508 [cs] (2018)

6. Jia, S., Despinasse, A., Wang, Z., Delingette, H., Pennec, X., Jaïs, P., Cochet, H., Sermesant, M.: Automatically Segmenting the Left Atrium from Cardiac Images Using Successive 3d U-Nets and a Contour Loss. In: Statistical Atlases and Computational Modeling of the Heart (STACOM) (Sep 2018)

7. Mahida, S., Sacher, F., Dubois, R., Sermesant, M., Bogun, F., Haïssaguerre, M., Jaïs, P., Cochet, H.: Cardiac Imaging in Patients With Ventricular Tachycardia. Circulation 136(25) (2017)

8. Mitchell, C.C., Schaeffer, D.G.: A two-current model for the dynamics of cardiac membrane. Bulletin of Mathematical Biology 65(5), 767-793 (Sep 2003)

9. Prakosa, A., Arevalo, H.J., Deng, D., Boyle, P.M., Nikolov, P.P., Ashikaga, H., Blauer, J.J.E., Ghafoori, E., Park, C.J., Blake, R.C., Han, F.T., MacLeod, R.S., Halperin, H.R., Callans, D.J., Ranjan, R., Chrispin, J., Nazarian, S., Trayanova, N.A.: Personalized virtual-heart technology for guiding the ablation of infarctrelated ventricular tachycardia. Nature Biomedical Engineering p. 1 (Sep 2018) 
10. Yamashita, S., Sacher, F., Hooks, D.A., Berte, B., Sellal, J.M., Frontera, A., Al Jefairi, N., Komatsu, Y., Amraoui, S., Denis, A., Derval, N., Sermesant, M., Laurent, F., Montaudon, M., Hocini, M., Haïssaguerre, M., Jaïs, P., Cochet, H.: Myocardial wall thinning predicts transmural substrate in patients with scar-related ventricular tachycardia. Heart Rhythm 14(2), 155-163 (Feb 2017)

11. Yamashita, S., Sacher, F., Mahida, S., Berte, B., Lim, H.S., Komatsu, Y., Amraoui, S., Denis, A., Derval, N., Laurent, F., Sermesant, M., Montaudon, M., Hocini, M., Haïssaguerre, M., Jaïs, P., Cochet, H.: Image Integration to Guide Catheter Ablation in Scar-Related Ventricular Tachycardia. Journal of Cardiovascular Electrophysiology 27(6), 699-708 (2016)

12. Yezzi, A., Prince, J.: An eulerian PDE approach for computing tissue thickness. IEEE Transactions on Medical Imaging 22(10), 1332-1339 (Oct 2003) 